\title{
NUEVA EVIDENCIA EMPÍRICA SOBRE EL EMPAREJAMIENTO LABORAL EN EL MERCADO DE TRABAJO ANDALUZ: LAS VACANTES
}

\author{
Fernando Núñez*, Daniel Otoł, Carlos Usabiaga ${ }^{1}$ \\ *Universidad de Sevilla; $\uparrow$ Universidad Pablo de Olavide
}

Recibido el 30 de mayo de 2010; aceptado el 19 de mayo de 2011

\section{Resumen}

En este artículo se analiza el proceso de emparejamiento en el mercado de trabajo andaluz a partir de una encuesta sobre empresas. Más concretamente, estudiamos la influencia de una serie de variables relevantes en el proceso de cobertura de las vacantes. Para ello, se han estimado modelos de duración para la tasa de salida de las vacantes. Cabe destacar, entre otros resultados, el predominio de las redes sociales como cauce para cubrir las vacantes. También se observa la falta de correspondencia entre la frecuencia de uso de los cauces y su eficacia. Así, las empresas privadas de intermediación laboral son el cauce menos utilizado, a pesar de ser el más rápido para cubrir casi todos los tipos de vacantes. Palabras clave: Andalucía, emparejamiento laboral, vacantes, redes sociales, datos individuales.

Clasificación JEL: J63, J64.

\begin{abstract}
This work analyses the matching process in the Andalusian labour market using a survey on companies. More specifically, we focus on the influence of a series of relevant variables in the process of coverage of the vacancies. For that purpose, duration models have been estimated for the hazard rate of the vacancies. It is necessary to emphasise, among other results, the importance of the social networks as a way to cover the vacancies. In addition, we also conclude that the correspondence between the frequency of use and the efficiency of the different channels does not exist. Thus, private intermediaries are, at the same time, the least used channel and the fastest to cover almost all types of vacancies.
\end{abstract}

Key words: Andalusia, labour matching, vacancies, social networks, individual data.

JEL Classification: J63, J64.

\footnotetext{
1 Dirección para correspondencia: cusaiba@upo.es. Agradecemos las sugerencias recibidas de Pablo Álvarez de Toledo, José María Arranz, J. Ignacio García-Pérez, Miguel Ángel Malo, Yolanda Rebollo, dos evaluadores de la Revista de Economía Laboral y los participantes en el XIII Encuentro de Economía Aplicada (Sevilla, 2010). Por supuesto, los errores que persistan son de nuestra entera responsabilidad. También agradecemos la financiación recibida de las siguientes instituciones: Ministerio de Ciencia e Innovación (ECO 2009-13357) y Junta de Andalucía (Proyectos de Excelencia (CEIC): P07-SEJ-02905, P09-SEJ-4546; Centro de Estudios Andaluces: PRY 027/08, PRY 010/10; Plan Andaluz de Investigación: SEJ-246, TEP-216).
}

(C) Revista de Economía Laboral 


\section{Introducción}

Los estudios del mercado de trabajo que utilizan modelos de búsqueda y emparejamiento han tenido una creciente importancia en las últimas décadas ${ }^{2}$. Según estos modelos, el emparejamiento laboral debe entenderse como un proceso sometido a fricciones que generan unos costes para los trabajadores y para las empresas. Como escribe Samuel Bentolila al comentar el Premio Nobel de Economía (2010) concedido a Diamond, Mortensen y Pissarides:

"Buscar empleo suele implicar actividades como preguntar a parientes, amigos y conocidos, ir a los servicios públicos de empleo, enviar currículos a empresas, apuntarse en empresas de trabajo temporal $y$ en bolsas de trabajo en internet, $y$ preparar $y$ tener muchas entrevistas de trabajo (y pasar el trago). Cubrir una vacante suele implicar anunciarla entre los empleados de la empresa, contratar a una empresa de selección, entrevistar a varios candidatos (a menudo con cierta frustración), evaluarlos y hacer ofertas. Las partes deben calcular si el emparejamiento entre ambas será mutuamente beneficioso, a veces (si una de las partes no tiene todo el poder de negociación) negociar las condiciones (salarios, jornada, tipo de contrato, etc.) y decidir si no sería mejor esperar a encontrar un mejor emparejamiento. Los economistas denominamos "fricciones" a los costes derivados de todas estas actividades". ${ }^{3}$

La literatura empírica sobre la búsqueda y el emparejamiento en el mercado de trabajo se ha orientado en la última década fundamentalmente hacia el empleo de bases de datos desagregadas paneles de datos y bases de datos individuales- que han permitido, entre otras cosas, realizar contrastes sobre el modelo teórico, controlar por heterogeneidades $\mathrm{y}$ fricciones, $\mathrm{y}$ conocer determinados aspectos fundamentales del proceso de emparejamiento, como la probabilidad estimada de encontrar un empleo atendiendo al perfil del desempleado. Dentro de esta línea econométrica de análisis con datos individuales se

\footnotetext{
2 Algunos trabajos que nos pueden servir de referencia sobre esta amplia e importante literatura son los siguientes: Blanchard y Diamond (1992), Coles y Smith (1998), Mortensen y Pissarides (1999), Pissarides (2000), Petrongolo y Pissarides (2001), Shimer (2007, 2010), Mortensen (2009) y Ebrahimy y Shimer (2010).

${ }^{3}$ Cita procedente del artículo "La Teoría de la Búsqueda Encuentra al Nobel", publicado en el blog Nada es Gratis, de la Fundación de Estudios de Economía Aplicada (FEDEA). Fecha de publicación: 12/10/2010. (http://www.fedeablogs.net/economia).
} 
engloba nuestro trabajo; en concreto, empleamos la técnica de los modelos de duración para estimar las tasas de salida de los puestos vacantes hacia el emparejamiento en el mercado de trabajo andaluz.

La disponibilidad de datos de duración es realmente útil cuando se trata de analizar fenómenos económicos en los que el tiempo es en sí mismo el factor que suscita el interés de los investigadores; por ejemplo, pensemos en la duración de una huelga, la duración de un episodio de desempleo, la supervivencia de un negocio, etc. ¿De qué dependen estas duraciones? ¿Cuál es la probabilidad de que se produzca el evento que haga concluir el episodio estudiado? ¿De qué depende esta probabilidad? La mejor forma de analizar este tipo de fenómenos es disponer de datos individuales con la historia de cada agente en un determinado periodo; es decir, con las fechas de entrada y salida en un determinado estado -el desempleo, por ejemplo- en dicho periodo. Sin embargo, los datos sobre duraciones presentan una serie de características, como la existencia de censura o de variables explicativas que cambian a lo largo de la duración del individuo, que crean problemas de estimación con los métodos estándares tales como la regresión múltiple -véase Jenkins (2005, cap.1)-. En este sentido, la técnica de los modelos de duración o supervivencia, que empleamos en este trabajo, permite tratar adecuadamente tales características de los datos generando estimaciones sin sesgos.

En la literatura de los modelos de duración aplicados al emparejamiento laboral es frecuente la estimación de tasas de salida para los trabajadores en situación de búsqueda de empleo; estimaciones en las que se trata de determinar la influencia en la duración de determinadas covariables, como el sexo, la edad, la experiencia laboral, la educación, la disposición a la movilidad geográfica, las prestaciones por desempleo, determinados programas o subsidios, y variables macroeconómicas como la tasa de paro. En España, en este campo, se ha prestado especial atención al papel de las prestaciones por desempleo y a la inserción laboral de los jóvenes ${ }^{4}$. Una línea de investigación muy importante en este campo es la que estudia salidas alternativas de la situación de búsqueda de empleo. Por ejemplo, en el caso español, se ha prestado especial atención al tránsito a contrato permanente frente a contrato temporal, y al autoempleo frente al trabajo por cuenta ajena ${ }^{5}$. Sin embargo, son muy

\footnotetext{
4 Existe un amplio desarrollo bibliográfico sobre modelos de duración aplicados al mercado de trabajo español. Así, a modo de muestra, podemos destacar los trabajos de Ahn y Ugidos (1995), Ahn et al. (1999), Bover et al. (2002), Jenkins y García-Serrano (2004), García-Pérez (2006) y Güell y Hu (2006). Para el caso andaluz, véanse Congregado y García-Pérez (2002) y Gámez y García-Pérez (2004).

5 Respecto al tránsito desde la situación de búsqueda a diferentes opciones, podemos recordar, por ejemplo, los trabajos de Carrasco (1999), Bover y Gómez (2004), Alba-
} 
poco frecuentes en nuestro país los modelos de duración aplicados a los puestos vacantes -véanse Álvarez de Toledo et al. (2011a, 2011b)-, ya que la disponibilidad de datos es bastante más reducida, tratándose de datos correspondientes a las oficinas públicas de empleo o a segmentos laborales específicos (bolsas universitarias, colegios profesionales, etc.). De hecho, para el caso español, muy pocos trabajos han prestado atención al análisis de las vacantes desde los trabajos de Antolín de mediados de los años noventa. En nuestro análisis estudiaremos las duraciones de los puestos vacantes correspondientes al conjunto de la economía andaluza; es decir, sin restringirnos a un segmento laboral concreto.

La base de datos que hemos utilizado es una encuesta encargada en el año 2008 por el Instituto de Desarrollo Regional (IDR), fundación universitaria adscrita a la Universidad de Sevilla. El fin de la encuesta era suministrar información para el proyecto Costes Laborales, Productividad y Empleabilidad en el Mercado de Trabajo Andaluz, financiado por el Servicio Andaluz de Empleo (SAE) y dirigido por Carlos Usabiaga. En la encuesta se incluían algunas preguntas secundarias referidas a la problemática del emparejamiento en el mercado de trabajo andaluz. Dado que el citado proyecto se concentró en otras variables, la información contenida en esas preguntas no llegó a explotarse; de ahí la idea de utilizarlas ahora para el análisis del emparejamiento en el mercado laboral andaluz.

En cuanto a la motivación de nuestro trabajo, aparte de lo ya apuntado respecto al análisis de las vacantes, creemos innecesario insistir en un problema tan importante como es el diferencial de desempleo andaluz ${ }^{6}$. Según la Encuesta de Población Activa (EPA), la tasa de paro andaluza se situó en torno al $28 \%$ en el año 2010, lo que coloca a esta Comunidad Autónoma a la cabeza -junto con Canarias- de las regiones españolas más azotadas por el desempleo. Pensamos que el análisis del emparejamiento laboral en esta región puede arrojar luz sobre ese problema persistente, al menos parcialmente.

El resto del artículo se organiza en cuatro secciones. En la siguiente sección desarrollamos el marco teórico de nuestro análisis empírico. En la sección 3 analizamos la encuesta utilizada y presentamos los principales resultados de la estadística descriptiva. La sección 4 se centra en el análisis de la tasa de salida de los puestos vacantes mediante la metodología Kaplan-Meier y mediante modelos de duración. Por último, en la sección 5 se exponen las principales conclusiones de nuestro trabajo.

Ramírez et al. (2007), Güell y Petrongolo (2007), Albert et al. (2008) y Arranz et al. (2010).

${ }^{6}$ Véanse Jimeno y Bentolila (1998), Elhorst (2003) y Usabiaga (2004). 


\section{Marco teórico}

Los modelos de búsqueda y emparejamiento en el mercado de trabajo se caracterizan principalmente porque introducen "fricciones" en el proceso de búsqueda de una pareja laboral, que lo alejan de ser un proceso donde oferta y demanda de trabajo se casan de forma rápida. La existencia de información imperfecta y de heterogeneidad entre los trabajadores y entre los puestos de trabajo son las dos principales fricciones consideradas en este tipo de modelos, las cuales hacen que el mercado de trabajo walrasiano tenga poca verosimilitud. Así, más que contactar en mercados centralizados donde el empleo y los salarios son determinados por la interacción de la oferta y la demanda de trabajo, los trabajadores y las empresas contactan entre sí en un mercado descentralizado, involucrándose en un lento y costoso proceso para emparejar preferencias específicas y cualificaciones. Esta dinámica del emparejamiento puede tener implicaciones sobre la forma en que el empleo y los salarios responden a las perturbaciones de carácter económico -véanse Pissarides (2000) y Shimer (2010)-.

La ecuación fundamental del modelo de búsqueda y emparejamiento es la llamada función agregada de emparejamiento laboral, que proporciona en su expresión más simple el flujo de colocaciones $(M)$ en cada periodo en función de los stocks iniciales de buscadores de empleo -en su mayoría desempleados- $(U)$ y de puestos de trabajo -en su mayoría puestos vacantes- $(V)$ :

$$
M=M(U, V)
$$

La función de emparejamiento puede expresarse, de modo secuencial, como el producto de dos factores: una función que determina el flujo total de contactos entre las empresas con puestos vacantes y los demandantes de empleo que envían solicitudes a dichas empresas, que llamaremos función de encuentro o contacto $C(U, V)$; y la fracción de dichos contactos $(\Phi)$ que acaban dando lugar a una contratación, llamada tasa de aceptación, que dependerá de la naturaleza y del grado de heterogeneidad existente en el mercado de trabajo.

$$
M(U, V ; \Phi)=\Phi C(U, V)
$$


Si suponemos inicialmente exógena la tasa de aceptación $(\Phi)$, el comportamiento atribuido a la función de emparejamiento dependerá de las propiedades de la función de contacto ${ }^{7}$.

La función de emparejamiento de la ecuación (2) representa un proceso de naturaleza agregada donde la heterogeneidad existente en el mercado de trabajo no se observa de forma explícita; pero es posible ampliar dicha función para controlar algunos tipos de heterogeneidad. En concreto, si nos centramos en los puestos vacantes que ofrecen las empresas, podemos controlar por heterogeneidad al menos de dos formas: haciendo endógena la tasa de aceptación e introduciendo intensidad diferencial en la búsqueda que lleva a cabo la empresa.

Así, podemos endogeneizar la tasa de aceptación de un candidato por parte de la empresa a través de su dependencia de la productividad de reserva del puesto. En el mercado laboral los trabajadores muestran distintas productividades y las empresas contratarán a un trabajador cuando su productividad supere a la mínima requerida para el puesto, que dependerá positivamente del coste salarial. La búsqueda de un trabajador adecuado tiene beneficios esperados para la empresa, ya que incrementa la probabilidad de encontrar a un trabajador que se ajuste a sus requerimientos, pero también tiene costes, tanto explícitos -por ejemplo, todos los costes asociados al proceso de selección- como implícitos -la búsqueda conlleva un coste de oportunidad, medido en términos del mejor candidato rechazado-. En este sentido, podemos suponer que cuando la empresa $i$ contacta con un trabajador obtiene una productividad $(p)$ extraída de una distribución de probabilidad $G(p)$. Si dicha distribución es conocida por las partes, la política óptima de la empresa consistirá en la elección de una productividad de reserva $\left(P_{i}\right)$ tal que el trabajador será contratado si $p \geq P_{i}$ y rechazado en otro caso, de modo que la probabilidad de que la empresa contrate a un candidato vendrá dada por $\Phi\left(P_{i}\right)=1-G\left(P_{i}\right)$.

Otra fuente de heterogeneidad en el emparejamiento por el lado de la empresa se vincula a la intensidad individual de búsqueda de candidatos -pensemos por ejemplo en el número de canales de búsqueda que puede emplear la empresa para dar a conocer un puesto vacante-. Así, la probabilidad de ser contactada por un trabajador de una empresa que emplea por periodo $s_{i}$ unidades de búsqueda para cada puesto vacante vendrá dada por $s_{i} / s C(U, s V) / V$, donde $s$ representa el promedio de unidades de búsqueda por puesto vacante y entra de forma multiplicativa en la función de contacto. Las unidades de búsqueda son ofrecidas a un determinado coste, que es normalmente creciente, y son elegidas de

\footnotetext{
${ }^{7}$ Normalmente, se asume en la literatura teórica que dichas funciones son crecientes en
} $U$ y $V$, cóncavas hacia el origen y con rendimientos a escala constantes. 
manera óptima por la empresa para maximizar el rendimiento neto de la búsqueda. Por tanto, diferentes empresas escogerán distintos niveles de intensidad, dependiendo de su coste de búsqueda, del coste de tener el puesto sin cubrir y del rendimiento esperado de conseguir ocuparlo ${ }^{8}$.

Con todos estos elementos, podemos expresar la probabilidad $\left(\mu_{i}\right)$ de que un puesto vacante $i$ forme una colocación productiva como el producto de dos probabilidades ${ }^{9}$ : la probabilidad de contactar con un candidato, que depende fundamentalmente de la intensidad de búsqueda de las partes y de los niveles de $U$ y $V$; y la probabilidad de aceptarlo para el puesto $(\Phi)$, que depende esencialmente de la productividad de reserva del puesto:

$$
\mu_{i}=\Phi_{i} \frac{s_{i}}{s} \frac{C(U, s V)}{V}=\left[1-G\left(P_{i}\right)\right] \frac{s_{i}}{s} \frac{C(U, s V)}{V}
$$

La probabilidad de cobertura $\left(\mu_{i}\right)$ puede depender del tiempo $(t)$ que la empresa permanece buscando. Así, dicha probabilidad podría disminuir con el paso del tiempo por varias razones: si aumenta la congestión o competencia por el lado de las empresas oferentes de puestos, si la empresa ha revisado todo el stock de demandantes sin éxito, de modo que su probabilidad de emparejamiento se limita a la entrada de nuevos candidatos, o si la empresa disminuye su intensidad de búsqueda a medida que observa que no aparecen candidatos adecuados para el puesto. Sin embargo, también podrían buscarse argumentos que explicaran que la tasa de cobertura mostrase una dependencia positiva con la duración; por ejemplo, si aumentara el ratio $U / V$, o si la empresa revisase a la baja su productividad de reserva o mejorase las condiciones del puesto a medida que transcurre el tiempo de búsqueda.

Por tanto, resulta de interés estimar la tasa de salida o cobertura de un puesto vacante dependiendo de su duración $\left(\mu_{i}(t)\right)$; esto es, la probabilidad de que un puesto que ha permanecido vacante hasta el comienzo del periodo $t$, se empareje a lo largo de dicho periodo.

$$
\mu_{i}(t)=\Phi_{i}(t) \frac{s_{i}(t) C\left(U_{t}, s V_{t}\right)}{s V_{t}}=\left[1-G\left(P_{i}(t)\right)\right] \frac{s_{i}(t) C\left(U_{t}, s V_{t}\right)}{s V_{t}}
$$

\footnotetext{
8 Un análisis detallado sobre la intensidad de la búsqueda de empleo puede verse, por ejemplo, en Pissarides (2000, cap. 5).

9 Todo el razonamiento sería simétrico para el caso de un trabajador que busca empleo.
} 
Núñez et al. / Revista de Economía Laboral 8 (2011), 1-27

Aunque hemos obtenido la tasa de salida o cobertura de un puesto mediante el producto de las dos probabilidades citadas (probabilidad de contactar con un trabajador y probabilidad de aceptarlo), en este trabajo, dada la naturaleza de los datos de que disponemos, se hará un análisis de la forma reducida de dicha tasa; es decir, analizaremos los efectos netos de las variables disponibles sobre la tasa de cobertura de un puesto vacante, no pudiéndose estudiar el efecto diferenciado de nuestras variables explicativas sobre cada parámetro estructural de la ecuación (4).

\section{Descripción de los datos}

La encuesta utilizada consta de 806 entrevistas a empresas con sede en Andalucía ${ }^{10}$. Del cómputo total de empresas, nosotros nos centraremos en las 720 que ofrecen datos sobre vacantes. A cada empresa se le formuló una serie de cuestiones relacionadas con su actividad productiva, tales como el número de trabajadores, el volumen de negocio, etc. Además, se le preguntó sobre cuestiones referidas a los cauces utilizados y el tiempo necesario para cubrir diferentes tipos de vacantes.

El objeto básico de esta investigación es el "timing" del emparejamiento laboral; en consecuencia, la variable que va a centrar nuestra atención es la duración requerida para cubrir los puestos vacantes. Dichas duraciones son medidas en días.

A cada empresa de la muestra se le pregunta por la duración de cobertura de sus vacantes, atendiendo al tipo de contrato (vacantes de contrato indefinido o temporal) y al nivel de cualificación requerido (sin estudios, estudios primarios, estudios secundarios o estudios universitarios), y se le solicita información sobre los cauces que utiliza para cubrir cada tipo de vacante. Dado que todas las empresas no ofertan todos los tipos de vacantes, hay empresas que no ofrecen información para los seis tipos de vacantes (véase la última fila de datos del cuadro 1).

En síntesis, nuestra base de datos nos ofrece la siguiente información para cada vacante: la duración de cobertura, el cauce utilizado para su cobertura y las características de la empresa que informa sobre la vacante.

La exposición de esta sección la dividiremos en dos partes. Primero atenderemos a las características generales de las empresas y a su relación con la duración de cobertura de los distintos tipos de vacantes.

\footnotetext{
${ }^{10}$ La encuesta también ha sido dirigida a una muestra de la población activa andaluza, pero dicha información no ha sido utilizada para este trabajo ya que hemos considerado que este flanco es menos novedoso -véanse las referencias recogidas en la sección introductoria-
} 
Posteriormente, nos centraremos en los cauces de búsqueda utilizados para cubrir cada tipo de vacante y en la duración de cobertura asociada a cada cauce.

\subsection{Caracteristicas generales de las empresas.}

El cuadro 1 ofrece información sobre las características de las empresas (columna 2), y sobre la duración media y mediana de cobertura de cada tipo de vacante según el nivel de cualificación requerido para cubrirla (columnas 3 a 10) y según el tipo de contrato (columnas 11 a 14).

En la primera fila de datos se recoge la duración de cobertura para cada tipo de vacante sin filtrar por ninguna característica de la empresa. En cuanto a las vacantes según el nivel de formación requerido, las duraciones de cobertura parecen estar relacionadas positivamente con el nivel de cualificación del puesto. Entre los dos primeros niveles no se observan apenas diferencias, pero de primaria a secundaria, y de secundaria a nivel universitario, se incrementa la duración (en particular la duración media). En cuanto a los puestos vacantes según el tipo de contrato, los de contrato indefinido tardan de media más del doble en cubrirse que los puestos de contrato temporal $(41,3 \text { días frente a } 18,4)^{11}$; sin embargo, las duraciones medianas coinciden.

El tiempo de cobertura de los puestos vacantes, además de estar relacionado con el nivel de cualificación requerido y con el tipo de contrato, depende también de las características de las empresas, como se puede observar en el resto de filas del cuadro 1.

\footnotetext{
11 Este resultado discrepa con el que Álvarez de Toledo et al. (2011a) observan para las vacantes gestionadas por el SAE, donde los puestos que ofrecen un contrato indefinido duran la mitad que los de carácter temporal. Estas diferencias pueden deberse a las distintas características del proceso de emparejamiento según el ámbito laboral considerado (general vs. oficinas públicas).
} 
GRÁFICO 1

Histograma de la duración de cobertura de las vacantes

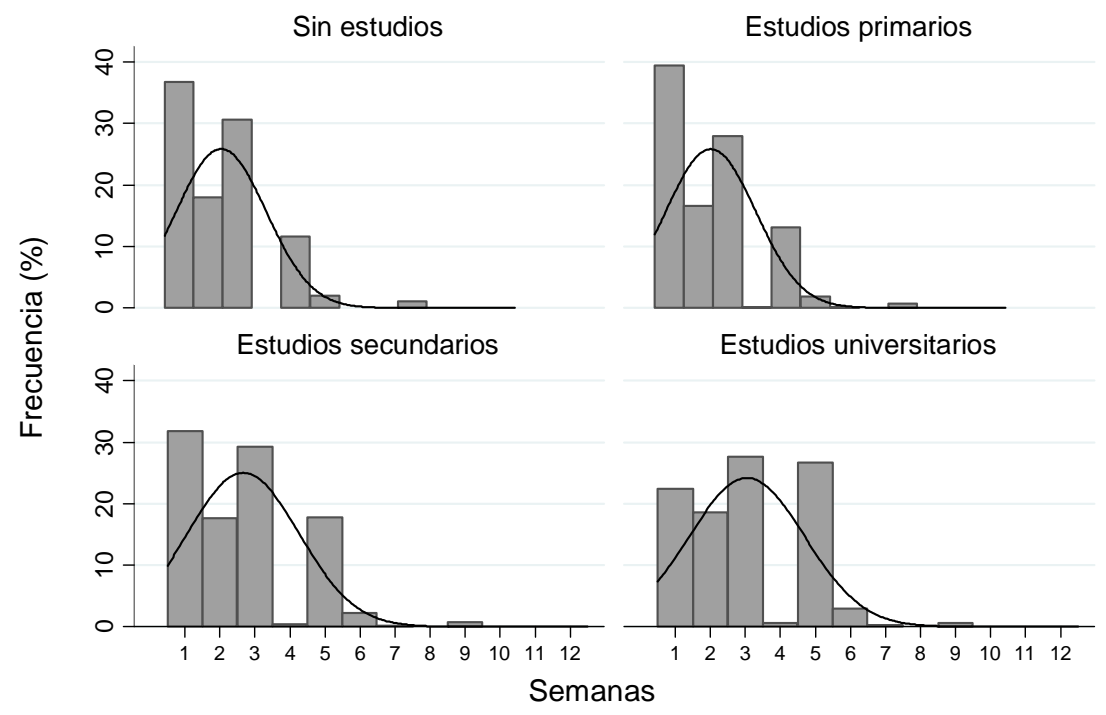

GRÁFICO 2

Histograma de la duración de cobertura de las vacantes

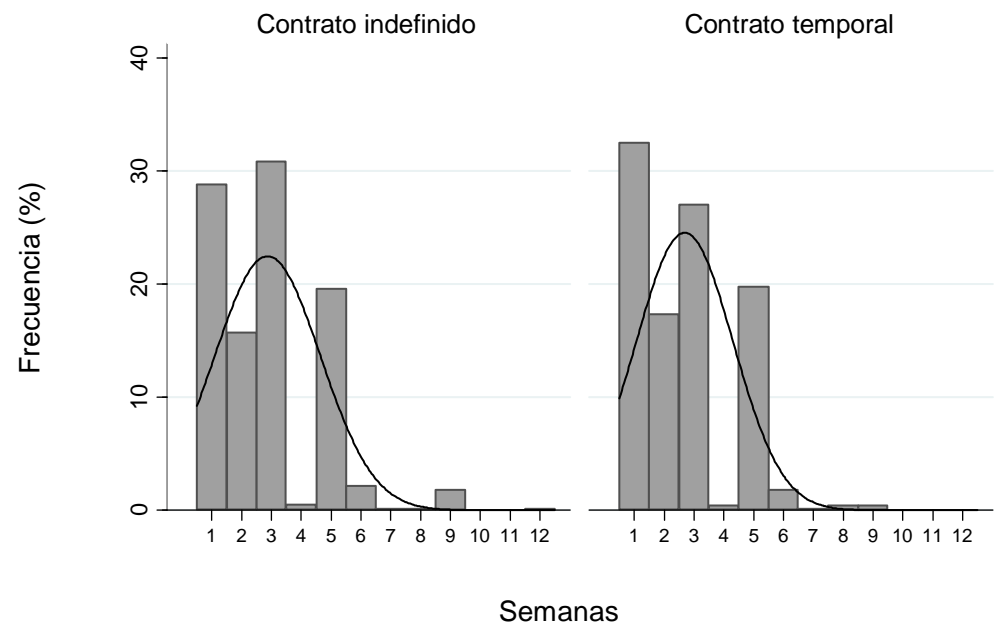


El gráfico 1 representa los cuatro histogramas de la duración de cobertura de un puesto dependiendo del nivel de cualificación. Dichos histogramas están agrupados en intervalos de 7 días, y limitados en la semana 12, ya que en dicho periodo se concentra la mayor parte de la información. En coherencia con sus valores medios, las duraciones para los niveles sin estudios y estudios primarios están más concentradas en las primeras semanas. En concreto, las dos primeras semanas representan en ambos niveles alrededor del 55\% de su distribución; sin embargo, ese mismo periodo sólo concentra el $48 \%$ y el $40 \%$ para las vacantes que requieren estudios secundarios y universitarios respectivamente. Por tanto, las empresas cubren los puestos de menor nivel de cualificación con mayor rapidez. También se aprecia que en las 6 primeras semanas se cubren aproximadamente el $98 \%$ de las vacantes para todos los niveles de cualificación.

De forma análoga al gráfico 1 , el gráfico 2 recoge los histogramas sobre el tiempo de cobertura de los puestos vacantes según el tipo de contrato. Dentro del periodo de las dos primeras semanas se cubre el $48 \%$ de los puestos de carácter temporal y el $40 \%$ de los puestos indefinidos; y en el periodo que comprende las 6 primeras semanas se cubren el $96 \%$ de las vacantes de contrato temporal y el $87 \%$ de las vacantes de contrato indefinido. Esa rapidez de cobertura de las vacantes podría encajar con que el proceso de emparejamiento estudiado responde al modelo teórico "stock-flow" (Coles y Smith, 2008), para el caso extremo de trabajadores "en cola" 12 .

\subsection{Cauces de búsqueda para la cobertura de las vacantes.}

En una pregunta de la encuesta, se le pide al informante que ordene, de mayor a menor prioridad, los cauces de búsqueda de trabajadores según el tipo de puesto vacante. De toda esta información nos hemos quedado únicamente con la referente al cauce de búsqueda principal (el de mayor prioridad). En el cuadro 2 se recoge para cada tipo

\footnotetext{
12 En ese marco, las vacantes que se ofrecen al mercado se cubren relativamente rápido, mientras que no ocurre lo mismo para los trabajadores. Álvarez de Toledo et al. (2008) llega a esa conclusión para los datos macroeconómicos de las oficinas públicas de empleo españolas. Álvarez de Toledo et al. (2011a) también coincide en el resultado, para una muestra de datos microeconómicos de las oficinas públicas de empleo andaluzas. Por último, Álvarez de Toledo et al. (2011b) vuelve a respaldar el resultado, para los datos microeconómicos de toda la población de las oficinas públicas andaluzas. Por otro lado, este último trabajo muestra la heterogeneidad de los segmentos laborales, definidos cruzando varias variables, en su comportamiento en el emparejamiento laboral desde el punto de vista de la duración.
} 
de vacante la distribución del cauce (principal) de búsqueda y la duración media y mediana de cobertura asociada a cada cauce. Se observa en el cuadro que hubo un cierto número de informantes que eludieron responder a esta pregunta, lo cual reduce el número de observaciones disponibles para cada tipo de vacante.

En cuanto al nivel de uso, las redes sociales representan el canal de búsqueda más importante para todos los tipos de vacantes, oscilando su porcentaje entre el $24,9 \%$ y el $42,7 \%$. En segundo lugar se encuentran, a cierta distancia, las oficinas públicas de empleo, cuyo porcentaje de uso se sitúa alrededor del 20\%. En último lugar se encuentran las empresas privadas de intermediación laboral, cuya utilización como cauce de búsqueda ronda tan sólo el $5 \%{ }^{13}$.

Si nos centramos en las duraciones asociadas a cada canal de búsqueda, observamos que los intermediarios privados constituyen el cauce más rápido, excepto para el nivel superior de estudios. Es de destacar el buen lugar que ocupan las oficinas públicas en la clasificación, pues siempre se hallan en el segundo puesto en cuanto a la rapidez. Este buen resultado de las oficinas públicas quizás se deba al uso directo que de este cauce hacen las administraciones públicas, que por lo general ofrecen puestos que se cubren con relativa rapidez.

Resultan llamativos los resultados obtenidos para los intermediarios privados. Hemos observado que este cauce, a pesar de la rapidez que muestra, es el menos utilizado. Seguramente haya razones que expliquen esta discrepancia, como el perfil del trabajador que se consigue a través de cada cauce, el precio al que se obtiene, o vinculadas a factores macroeconómicos como la tasa de temporalidad o de paro. En cuanto a ese último factor, podría argumentarse que en un marco de elevada tasa de paro - como es el caso en Andalucía- es más fácil encontrar candidatos para cubrir un puesto vacante usando cauces informales, lo que haría menos necesaria la utilización del cauce de los intermediarios privados. Otro factor adicional que puede estar influyendo es el intervencionismo del sector público en el mercado laboral andaluz. Por

13 Una encuesta realizada por el Instituto de Estadística de Andalucía (IEA) en el año 1999 (Encuesta a Empresas sobre Tendencias del Mercado Laboral) también indica un elevado uso de las redes sociales (tabla 4.2.1). Así, según los resultados de dicha encuesta, el 58\% de las empresas andaluzas utiliza preferentemente los contactos personales, el 15,4\% los servicios públicos de empleo, el 8,2\% las empresas privadas de intermediación laboral, y, finalmente, el 18,4\% otros cauces. Por su parte, en otra encuesta del IDR (Encuesta sobre Modelos de Búsqueda de Empleo en Andalucía, 2005), se obtiene también que el cauce de las redes sociales supone la vía predominante de acceso al empleo. En concreto, un 46\% de los trabajadores reconoció haber accedido a su empleo mediante este cauce. 
otra parte, la reciente reforma laboral (2010) ha permitido la figura de los intermediarios privados con ánimo de lucro, factor que puede alterar en el futuro el comportamiento y peso de este cauce de búsqueda.

\begin{tabular}{|c|c|c|c|}
\hline \multicolumn{4}{|l|}{$\begin{array}{ll}\text { CUADRO } 2 \\
\end{array}$} \\
\hline \multirow{2}{*}{$\begin{array}{c}\text { Principal cauce de búsqueda utilizado para la cobertura de los } \\
\text { puestos vacantes }\end{array}$} & \multirow{2}{*}{$\begin{array}{c}\text { Distribución } \\
\text { porcentual }\end{array}$} & \multicolumn{2}{|c|}{$\begin{array}{l}\text { Duración de cobertura de } \\
\text { las vacantes (días) }\end{array}$} \\
\hline & & Media & Mediana \\
\hline \multicolumn{4}{|l|}{ Según el nivel de cualificación requerido: } \\
\hline \multicolumn{4}{|l|}{ Puestos vacantes: sin estudios (364 observaciones) } \\
\hline Oficinas públicas de empleo & $17,6 \%$ & 10,7 & 7 \\
\hline Empresas privadas de intermediación & $1,7 \%$ & 9,2 & 8,5 \\
\hline Portales de empleo de internet & $5,0 \%$ & 13,8 & 10 \\
\hline Red de contactos sociales y familiares & $35,7 \%$ & 15,5 & 10 \\
\hline Otros & $8,0 \%$ & 12,2 & 10 \\
\hline -Respuestas nulas & $32,1 \%$ & - & - \\
\hline \multicolumn{4}{|l|}{ Puestos vacantes: estudios primarios (553 observaciones) } \\
\hline Oficinas públicas de empleo & $22,6 \%$ & 12,1 & 7 \\
\hline Empresas privadas de intermediación & $5,4 \%$ & 4,5 & 2 \\
\hline Portales de empleo de internet & $6,7 \%$ & 12,8 & 10 \\
\hline Red de contactos sociales y familiares & $42,7 \%$ & 14,4 & 10 \\
\hline Otros & $11,9 \%$ & 14,9 & 15 \\
\hline -Respuestas nulas & $10,7 \%$ & - & - \\
\hline \multicolumn{4}{|l|}{ Puestos vacantes: estudios secundarios (582 observaciones) } \\
\hline Oficinas públicas de empleo & $22,3 \%$ & 12,1 & 8,5 \\
\hline Empresas privadas de intermediación & $6,2 \%$ & 9,8 & 5 \\
\hline Portales de empleo de internet & $10,7 \%$ & 20,9 & 15 \\
\hline Red de contactos sociales y familiares & $39,9 \%$ & 18,7 & 15 \\
\hline Otros & $15,5 \%$ & 14,9 & 15 \\
\hline -Respuestas nulas & $5,5 \%$ & - & - \\
\hline \multicolumn{4}{|l|}{ Puestos vacantes: estudios universitarios (309 observaciones) } \\
\hline Oficinas públicas de empleo & $21,4 \%$ & 18,4 & 10 \\
\hline Empresas privadas de intermediación & $10,0 \%$ & 22,8 & 20 \\
\hline Portales de empleo de internet & $10,4 \%$ & 23,8 & 25 \\
\hline Red de contactos sociales y familiares & $24,9 \%$ & 18,3 & 15 \\
\hline Otros & $13,6 \%$ & 18,6 & 15 \\
\hline -Respuestas nulas & $19,7 \%$ & - & - \\
\hline \multicolumn{4}{|l|}{ Según el tipo de contrato: } \\
\hline \multicolumn{4}{|l|}{ Puestos vacantes: contrato temporal (678 observaciones) } \\
\hline Oficinas públicas de empleo & $20,5 \%$ & 9,2 & 7 \\
\hline Empresas privadas de intermediación & $3,1 \%$ & 7,2 & 5 \\
\hline Portales de empleo de internet & $7,7 \%$ & 19,0 & 15 \\
\hline Red de contactos sociales y familiares & $40,0 \%$ & 15,4 & 15 \\
\hline Otros & $12,4 \%$ & 17,1 & 15 \\
\hline -Respuestas nulas & $16,4 \%$ & - & - \\
\hline \multicolumn{4}{|l|}{ Puestos vacantes: contrato fijo (669 observaciones) } \\
\hline Oficinas públicas de empleo & $16,4 \%$ & 18,4 & 10 \\
\hline Empresas privadas de intermediación & $5,4 \%$ & 12,0 & 10 \\
\hline Portales de empleo de internet & $7,9 \%$ & 19,3 & 15 \\
\hline Red de contactos sociales y familiares & $38,3 \%$ & 19,6 & 15 \\
\hline Otros & $10,3 \%$ & 21,6 & 15 \\
\hline -Respuestas nulas & $21,7 \%$ & - & - \\
\hline
\end{tabular}




\section{La tasa de salida de los puestos vacantes}

En esta sección se analiza la tasa de salida de los puestos vacantes. En primer lugar, realizamos un análisis no paramétrico, mediante la metodología Kaplan-Meier, y, en segundo lugar, estimamos un modelo de duración que nos permitirá controlar por ciertas características de los puestos.

\subsection{Análisis no paramétrico de la tasa de salida de las vacantes.}

El atractivo principal de la metodología Kaplan-Meier radica en la posibilidad de calcular el ritmo de salida de una situación dada y la probabilidad de permanecer en dicha situación conforme pasa el tiempo. La tasa de salida de una vacante mide la probabilidad de que ésta se cubra en un determinado periodo, dado que no se ha cubierto antes de ese periodo. La variable "duración de cobertura de las vacantes" contiene observaciones completas y exitosas. Este hecho se debe a que a las empresas se les preguntó sobre el tiempo que tardan en cubrir cada tipo de vacante, llevando implícita la pregunta el hecho de que la vacante se cubre $^{14}$. Para el análisis de las tasas de salida resulta conveniente censurar esta variable a partir de la décima semana, dado que más allá de este periodo se observan muchas discontinuidades en el histograma de frecuencias ${ }^{15}$. Por otra parte, hemos discretizado en periodos semanales la duración de cobertura de las vacantes, cuya unidad de medida temporal en la encuesta es el día, para corregir ciertas concentraciones de observaciones en determinados días.

\footnotetext{
14 El hecho de que todas las observaciones sean exitosas da lugar a tasas de salida sobreestimadas, pero pensamos que ello no afecta a nuestro análisis sobre el efecto relativo que cada covariable tiene en la tasa de salida.

15 En el periodo que comprende las 10 primeras semanas se cubren más del 95\% del total de vacantes.
} 
GRÁFICO 3

Tasa de salida de las vacantes por nivel de cualificación requerido

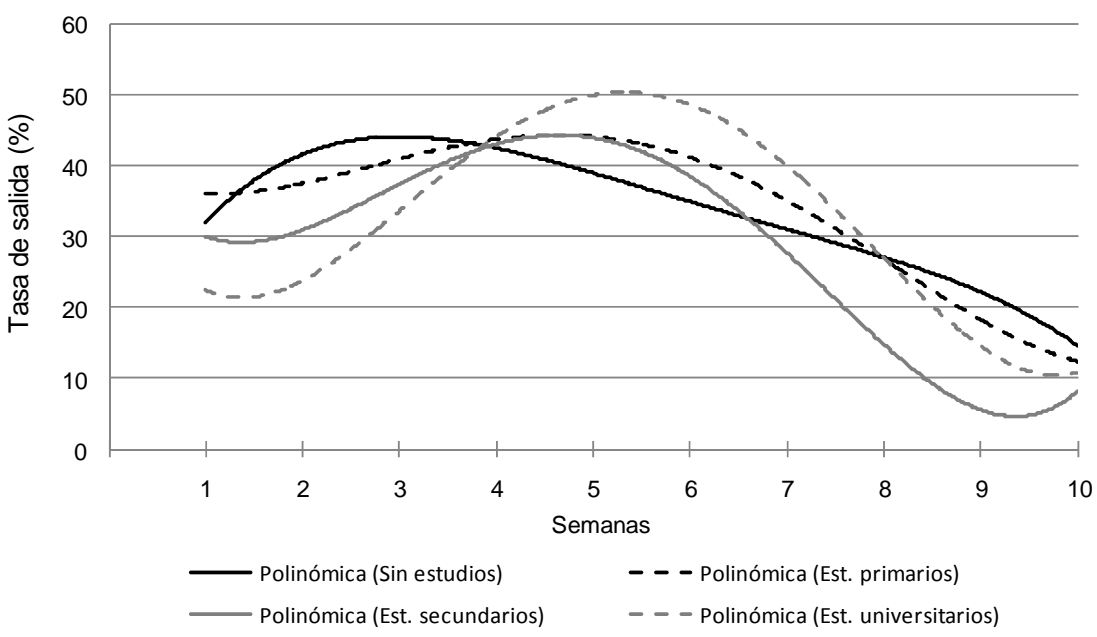

GRÁFICO 4

Tasa de salida de las vacantes por tipo de contrato

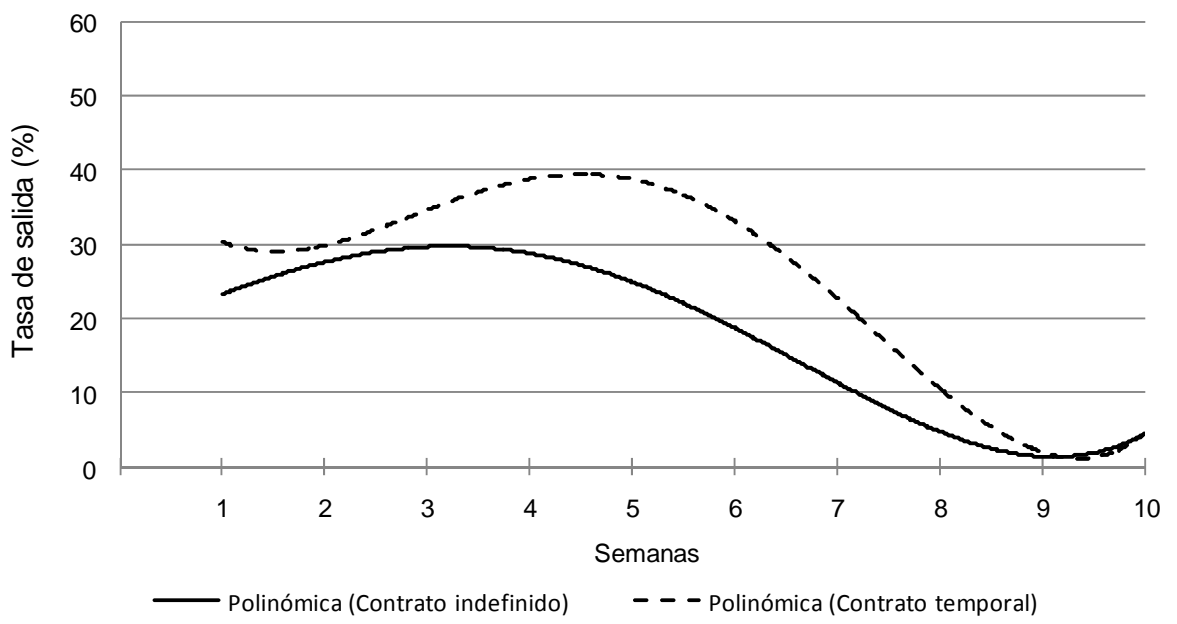

El gráfico 3 muestra la tasa de salida de las vacantes dependiendo del nivel de cualificación requerido para su cobertura. La tasa se ofrece alisada -mediante un polinomio de grado 4- para poder simplificar el análisis comparativo. Se observa que hasta la semana cuarta los puestos que requieren un mayor nivel de formación presentan una menor tasa de salida. El gráfico 4 es análogo al anterior, pero diferenciando entre las 
vacantes de contrato indefinido y las de contrato temporal. Se observa que la línea correspondiente a los puestos de carácter indefinido se sitúa por debajo (menor tasa de salida) de la línea de los puestos de carácter temporal. Finalmente, cabe destacar que en ambos gráficos se observa que las funciones de la tasa de salida son crecientes las primeras semanas, hasta llegar a un periodo a partir del cual empiezan a descender de manera pronunciada.

\subsection{Modelo de duración para la tasa de salida de las vacantes}

Hemos estimado un modelo de duración en tiempo discreto para la tasa de salida de las vacantes, utilizando un modelo "Complementary LogLog" de riesgos proporcionales ${ }^{16}$, el cual resulta adecuado en nuestro estudio fundamentalmente porque permite estudiar transiciones tomando el tiempo como una variable discreta y porque permite captar la dependencia con la duración de la tasa de salida. Dado que cada empresa proporciona varias observaciones de vacantes, hemos controlado por la posible correlación "intragrupo" que pudieran presentar los datos, obteniendo así estimaciones con errores estándar robustos.

Contamos con 720 empresas que ofrecen información sobre vacantes. En el cuestionario se pregunta a las empresas hasta por 6 tipos de vacantes diferentes -aunque no tienen por qué responder sobre todas-, de las cuales 4 tipos se refieren al nivel de formación requerido para el puesto y 2 tipos se refieren al tipo de contrato. Sin embargo, no tenemos vacantes con el cruce de ambas características. Este hecho provoca que no podamos estimar una sola regresión para la tasa de salida con todos los tipos de vacantes a la vez, sino que hay que estimar por separado dos regresiones, una según el nivel de cualificación, cuyos resultados se recogen en el cuadro 3 , y otra según el tipo de contrato, recogida en el cuadro 4.

Las variables que hemos introducido en la regresión para intentar explicar la tasa de salida de los puestos vacantes pueden clasificarse en 3 grupos. Un primer grupo corresponde a las dummies de duración, que se incluyen para analizar la influencia del tiempo en la tasa de salida. Un segundo grupo se refiere al tipo de vacante y al principal cauce de búsqueda. En las regresiones 1 y 2 de los cuadros 3 y 4 se incluyen ambas variables de manera separada, mientras que en las especificaciones 3 y 4 se introducen interacciones. En el tercer grupo se encuentran una serie de variables relacionadas con características generales de la empresa, que pensamos que pueden influir en la tasa de salida. Dentro de este grupo se

16 Véase Jenkins (2005, pág. 41). 
encuentran variables relacionadas con el tamaño de la empresa (número de trabajadores y facturación), con los trabajadores y las relaciones laborales (nivel de formación de los trabajadores de la empresa, porcentaje de indefinidos, salario medio, cursos de formación y horas de trabajo a la semana), y con otras características, como el año de constitución de la empresa (que puede servir de "proxy" para la experiencia empresarial) o la provincia a la que se vincula. El tercer grupo de variables sólo se incluye en las regresiones 2 y 4 de ambos cuadros.

Los resultados obtenidos muestran, en primer lugar, que de la semana 4 a la 6 aumenta la tasa de salida respecto al periodo de referencia (semanas 1-3), mientras que de la semana 7 a la 10 se reduce bruscamente. Este comportamiento se observa en todas las especificaciones de los cuadros 3 y 4 . El incremento inicial de la tasa de salida y el descenso en las últimas semanas resulta consistente con las funciones de la tasa de salida representadas en los gráficos 3 y 4 , y se podría vincular a que el proceso de emparejamiento en el mercado de trabajo andaluz se aproxima al descrito por el modelo "stock-flow", en el caso extremo de trabajadores "en cola"17. Este comportamiento de la tasa de salida aconsejaría articular políticas que hicieran más atractivas las vacantes difíciles de cubrir, así como darlas a conocer a todo el stock de demandantes de empleo ${ }^{18}$. A este respecto, podemos recordar el papel desempeñado por la mayor flexibilidad laboral del colectivo de los inmigrantes ${ }^{19}$.

17 En esencia, esto significa que el mercado laboral se caracteriza por la presencia persistente de un exceso de demandantes de empleo, casi todos ellos pertenecientes al "lado largo" del mercado, que compiten por las vacantes nuevas que se registran en cada periodo. Es decir, en unas pocas semanas, las nuevas vacantes se dan a conocer y se enfrentan al amplio stock de demandantes de empleo, cubriéndose en muchos casos; pero si las vacantes no se cubren a corto plazo -por ser poco atractivas, por presentar requerimientos complejos, por problemas administrativos, etc.- tienen que esperar para emparejarse a los nuevos demandantes de empleo que vayan entrando en el mercado, lo cual hace que su tasa de salida se reduzca. Véanse Álvarez de Toledo et al. (2008, 2011a).

18 Álvarez de Toledo et al. (2011a), respecto a las vacantes gestionadas por el SAE, proponen dar un tratamiento diferenciado a aquellos puestos de difícil cobertura, sugiriendo analizar las características de dichos puestos y revisar a fondo el territorio andaluz para encontrarles candidatos. Esta propuesta pensamos que es generalizable al conjunto de las vacantes que presentan dificultad para el emparejamiento.

19 Sobre la mayor flexibilidad laboral de los trabajadores inmigrantes puede consultarse Bentolila et al. (2008), donde se relaciona el aumento de la presencia de inmigrantes con una modificación, en sentido favorable, de la curva de Phillips de la economía española hasta la llegada de la crisis. Conde-Ruiz et al. (2009), tras estudiar diversas variables económicas, concluye que Andalucía pertenece al grupo de regiones más favorecidas por la inmigración hasta la llegada de la crisis. 
Respecto al nivel de formación requerido para cubrir un puesto, las especificaciones 1 y 2 del cuadro 3 muestran que la tasa de salida de las vacantes se reduce a medida que aumenta dicho requerimiento. En cuanto al tipo de contrato, el cuadro 4 muestra que las vacantes de contrato temporal se cubren antes que las de contrato indefinido (con una probabilidad un $25 \%$ superior). Sin embargo, los modelos con interacciones (regresiones 3 y 4) indican que el tipo de vacante (ya sea según formación o según tipo de contrato) no tiene un efecto uniforme sobre la tasa de salida, sino que dicho efecto está condicionado por el tipo de cauce que se utiliza para cubrir las vacantes. Por tanto, deben interpretarse los resultados teniendo en cuenta la interacción tipo de vacante-cauce de búsqueda. Para ello nos centraremos en la especificación 4, ya que incluye los términos de interacción y un mayor número de variables de control ${ }^{20}$.

En primer lugar, considerando a las vacantes que requieren un nivel de estudios inferior a secundaria, en el cuadro 3 se observa que el cauce de los intermediarios privados es el que presenta una mayor tasa de salida. El coeficiente indica que la tasa de salida de este tipo de vacante cuando se utiliza el cauce de las empresas privadas de intermediación laboral es un $69 \%$ superior a cuando se utiliza el cauce de las redes sociales. En segundo lugar, las vacantes que requieren estudios secundarios presentan una tasa de salida inferior con Internet (coeficiente: $0,75)$ que con las empresas privadas de intermediación (coeficiente: 1,09). También es posible apuntar que las vacantes de estudios secundarios cuyos cauces principales de búsqueda son Internet y las redes sociales muestran una tasa de salida inferior al grupo de referencia (vacantes con nivel de estudios inferior al secundario y que utilizan las redes sociales). En tercer lugar, en cuanto a las vacantes que requieren estudios universitarios, cabe destacar que para todos los cauces de búsqueda la tasa de salida es inferior al grupo de referencia, lo que resulta indicativo de que las vacantes con mayor nivel de estudios tardan más tiempo en cubrirse. Los tests de Wald de igualdad de coeficientes indican que, para el caso de las vacantes vinculadas a estudios universitarios, las redes sociales presentan una tasa de salida significativamente más elevada que las oficinas públicas, los intermediarios privados e Internet.

\footnotetext{
20 En el análisis de los términos de interacción nos centraremos sólo en aquellos coeficientes que presentan diferencias estadísticamente significativas entre ellos, de acuerdo con el test de Wald de igualdad de coeficientes.
} 
CUADRO 3

TASA DE SALIDA DE LAS VACANTES SEGÚN EL NIVEL DE CUALIFICACIÓN REQUERIDO ${ }^{1}$

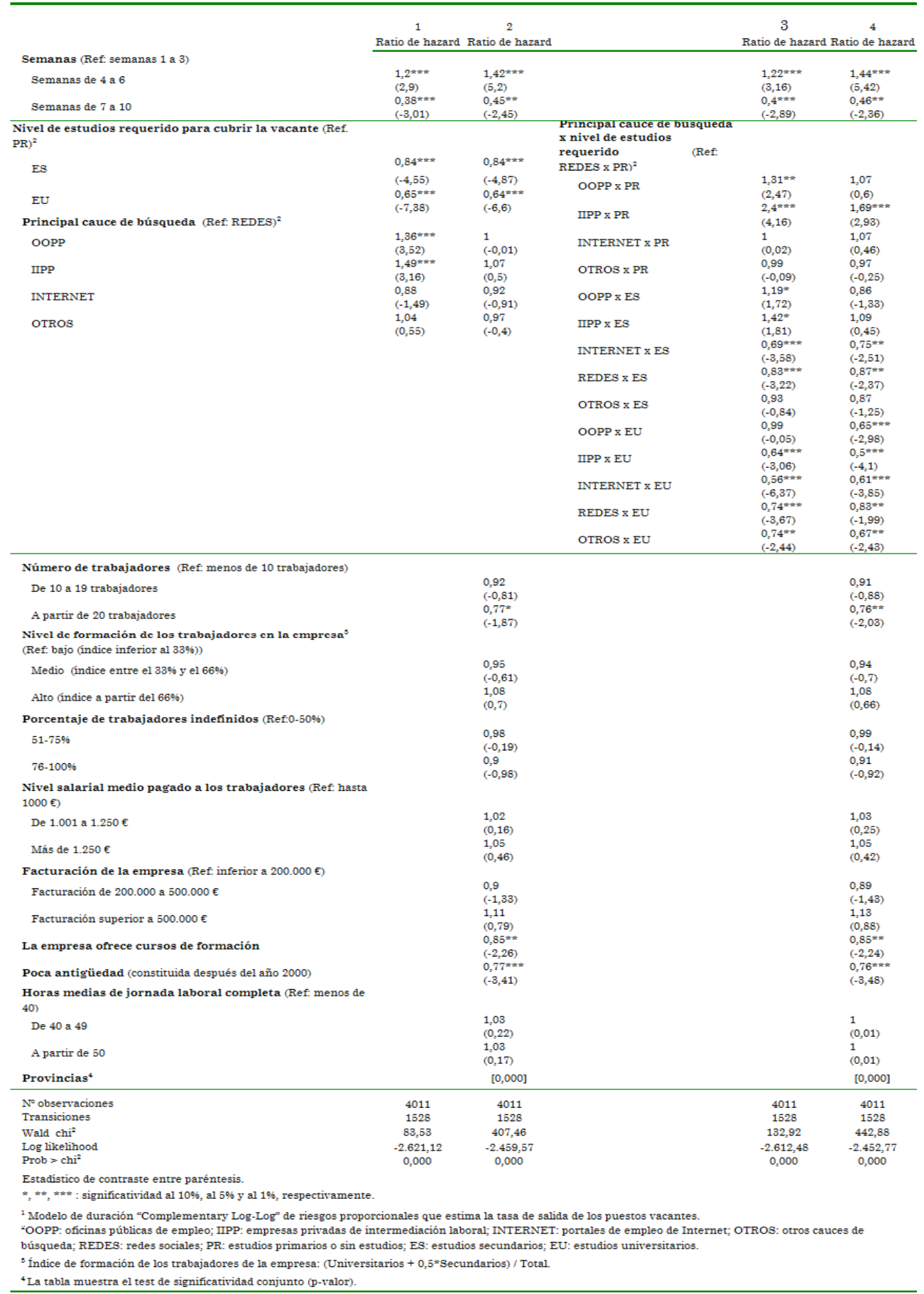


CUADRO 4

TASA DE SALIDA DE LAS VACANTES SEGÚN ELTIPO DE CONTRATO ${ }^{1}$

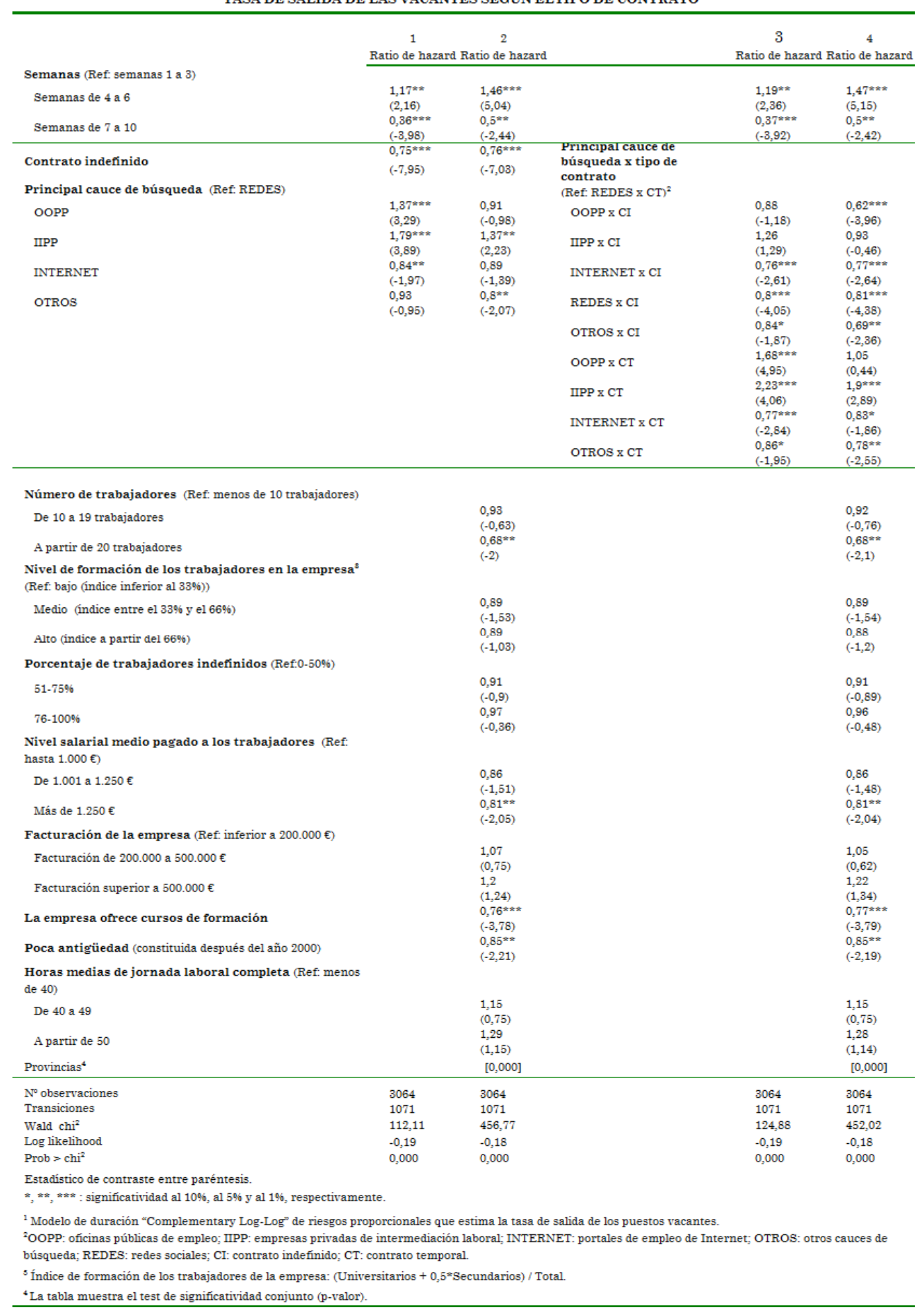


Respecto a la tasa de salida de las vacantes según el tipo de contrato (cuadro 4), se observa que, excepto en el caso del cauce de los intermediarios privados, las vacantes de contrato indefinido tienen una tasa de salida inferior al grupo de referencia (contrato temporal cuyo cauce son las redes sociales). Asimismo, entre las vacantes de carácter indefinido las oficinas públicas muestran un peor comportamiento que los intermediarios privados, Internet y las redes sociales. En lo que se refiere a las vacantes de contrato temporal, las empresas privadas de intermediación laboral constituyen el cauce con mayor tasa de salida (un 90\% superior al cauce de las redes sociales); mientras que las redes sociales y las oficinas públicas presentan tasas de salida superiores a Internet y al cauce residual ("otros"). Por último, se puede añadir que, para los cauces de las oficinas públicas, los intermediarios privados y las redes sociales, las vacantes de contrato temporal presentan una tasa de salida superior a las de contrato indefinido.

Pasando a considerar la influencia de las características de las empresas en la tasa de salida de las vacantes, se observa que las empresas con 20 o más trabajadores tienen una tasa de salida aproximadamente entre un $25 \%$ y un $30 \%$ inferior. Ello podría deberse quizás a que estas empresas de mayor tamaño implementan procedimientos de captación de trabajadores más complejos que las empresas más pequeñas, lo que puede aumentar la duración del proceso de cobertura de las vacantes.

Las empresas que ofrecen cursos de formación a sus trabajadores cubren sus vacantes con una tasa de salida inferior. Esa oferta formativa se podría considerar un indicador de la preocupación por el capital humano o del grado de complejidad de las tareas realizadas en la empresa, por lo que el resultado apuntado podría indicar que estas empresas se preocupan más por conseguir a los empleados adecuados.

La antigüedad de la empresa también parece ser un factor relevante. Así, la poca antigüedad (año de constitución posterior al año 2000) está relacionada con una menor tasa de salida de las vacantes.

A continuación, haremos balance de las evidencias obtenidas respecto a los cauces de cobertura de las vacantes, una variable central en nuestro análisis. En cuanto a la frecuencia de uso, el cauce preferido para cubrir las vacantes es el de las redes sociales; siendo el cauce menos usado los intermediarios privados. Sin embargo, resulta interesante observar que son precisamente los intermediarios privados el cauce que arroja en general duraciones de cobertura menores y tasas de salida superiores (excepto para las vacantes que requieren un nivel de estudios universitarios). Por el contrario, el cauce preferido (las redes sociales) presenta unos resultados bastante discretos en términos de duración y tasas de salida. 
Que los intermediarios privados sean el cauce menos usado y, al mismo tiempo, un cauce eficaz hace suponer que existen motivos para que este fenómeno, aparentemente contradictorio, ocurra. Como apuntamos en la sección 3, su escaso uso podría deberse quizás a un mayor coste para la empresa, a un menor nivel de calidad del emparejamiento (peores actitudes del trabajador, etc.), al intervencionismo del sector público, o estar vinculado a factores macroeconómicos como la elevada tasa de paro. Respecto a la rapidez del cauce, podría apuntarse como posible factor explicativo la escasa congestión de vacantes que presenta, al ser el menos utilizado. Por otro lado, el predominio de las redes sociales debe llevarnos a prestar atención a las posibles consecuencias que ello pudiera tener sobre el mercado de trabajo andaluz.

Los efectos de las redes sociales en este campo llevan tiempo siendo objeto de debate ${ }^{21}$. Como efectos positivos, se suele afirmar que las empresas pueden reducir los costes de búsqueda y de selección asociados al proceso de contratación gracias a la información obtenida mediante este cauce. Así ocurre, por ejemplo, con la búsqueda de candidatos recurriendo al círculo de conocidos de la plantilla de la empresa. Sin embargo, también deben tenerse en cuenta los posibles efectos negativos relacionados con el limitado ámbito en el cual la empresa busca candidatos, ámbito que viene marcado por los contactos sociales de los miembros de la organización. Además, se pueden citar como posibles consecuencias negativas los desajustes de cualificación, con efectos negativos sobre los salarios y la productividad $^{22}$, y el mantenimiento de las desigualdades sociales. Nuestros resultados también apuntan otra consecuencia negativa, pues la tasa de salida de las vacantes para las redes sociales es, en general, inferior a la de los intermediarios privados, por lo que el mayor uso relativo de dichas redes no favorece la agilidad del emparejamiento a nivel agregado. Por todo ello, debería prestarse mucha atención a la relevancia que presentan las redes sociales ${ }^{23}$ en el mercado de trabajo andaluz y a su

21 Entre los abundantes trabajos sobre "social networks" destacan los de CalvóArmengol (por citar algunos: Calvó-Armengol, 2004; y Calvó-Armengol y Matthew, 2004 y 2007).

${ }^{22}$ Bentolila et al. (2010) señalan que los contactos sociales, a pesar de poder reducir las duraciones de búsqueda, pueden presentar efectos negativos en términos de desajustes de cualificación, menores salarios y menor productividad agregada.

23 Hay que tener presente que hemos considerado como redes sociales a "la red de contactos sociales y familiares", ya que ésta es la definición recogida en el cuestionario. Sería muy interesante, de cara a profundizar en el tema, diferenciar los contactos familiares y sociales ajenos al ámbito profesional, de los contactos (sociales) puramente profesionales, ya que, como es evidente, estos últimos tienen un carácter muy distinto a los primeros. 
Núñez et al. / Revista de Economía Laboral 8 (2011), 1-27

relación con otros problemas económicos, laborales y sociales. En este sentido, para reducir esa dependencia, deberían fomentarse otros cauces de búsqueda, como los intermediarios privados y públicos o Internet ${ }^{24}$.

\section{Conclusiones}

El objetivo de este trabajo ha sido analizar las relaciones que se producen en el proceso de emparejamiento laboral de la economía andaluza. Gracias a la disponibilidad de una nueva encuesta sobre empresas, hemos podido estudiar variables que hasta la fecha han sido muy difíciles de valorar. Datos microeconómicos sobre vacantes que corresponden a todo el mercado laboral, y no sólo al segmento intervenido por el sector público, o a algunos sectores específicos, no son nada habituales, y hasta donde llega nuestro conocimiento no existen estudios equivalentes para España y sus regiones. Somos conscientes de que los datos utilizados presentan limitaciones, pero a la vez arrojan luz sobre algunos fenómenos que hasta la fecha han sido escasamente analizados. En cuanto a la metodología de nuestro trabajo, aparte del análisis de estadística descriptiva, hemos utilizado modelos de duración.

En general, se observan duraciones de cobertura de las vacantes bastante reducidas (pocas semanas). Nuestras estimaciones apuntan a que las empresas privadas de intermediación laboral consiguen en general una tasa de salida elevada, excepto para las vacantes que requieren un alto nivel de formación. Resulta llamativo que, sin embargo, las empresas privadas de intermediación sean el cauce menos usado para cubrir las vacantes, mientras que las redes sociales se colocan con diferencia en el primer lugar como cauce preferido. Consideramos que el predominio del uso de las redes sociales debe ser objeto de mucha atención, dados los posibles efectos adversos que podría estar generando en el mercado de trabajo andaluz, por diversas vías.

Sería interesante poder disponer de datos sobre otras Comunidades Autónomas y el conjunto de España, para poder comparar así nuestros resultados con los que pudieran registrarse en otras áreas. Cabría plantearse si la importancia de las redes sociales en Andalucía está relacionada con características autóctonas de este mercado de trabajo o si se trata de un fenómeno generalizable a todo el país. En este sentido, pensamos que la precariedad laboral, en términos de la alta tasa de desempleo y de temporalidad de la economía andaluza, es

\footnotetext{
24 En Andalucía, recientemente el SAE está potenciando la Oficina Virtual de Empleo, de manera que se pueden renovar las demandas, consultar ofertas, solicitar cursos de formación, etc., directamente por Internet.
} 
un factor determinante de nuestros resultados. También podríamos preguntarnos si los distintos cauces de emparejamiento laboral presentan efectos similares en todas las regiones españolas. Consideramos que estos interrogantes constituyen un campo fértil de investigación que sería interesante abordar.

\section{Bibliografía}

Ahn, N., De la Rica, S. y Ugidos, A. (1999): "Willingness to Move for Work and Unemployment Duration in Spain”, Economica, 66 (263), 335-357.

Ahn, N. y Ugidos, A. (1995): "Duration of Unemployment in Spain: Relative Effects of Unemployment Benefit and Family Characteristics", Oxford Bulletin of Economics and Statistics, 57 (2), 249-264.

Alba-Ramírez, A., Arranz, J. y Muñoz-Bullón, F. (2007): "Exits from Unemployment: Recall or New Job”, Labour Economics, 14 (5), 788-810.

Albert, C., Toharia, L. y Davia, M. (2008): "To Find or Not to Find a First "Significant" Job", Revista de Economía Aplicada, 16 (46), 37-59.

Álvarez de Toledo, P., Núñez, F. y Usabiaga, C. (2008): "La Función de Emparejamiento en el Mercado de Trabajo Español”, Revista de Economía Aplicada, 16 (48), 5-35.

Álvarez de Toledo, P., Núñez, F. y Usabiaga, C. (2011a): "An Empirical Analysis of the Matching Process in the Spanish Public Employment Agencies: The Vacancies", Universidad Pablo de Olavide, Department of Economics, Working Paper no 11-03.

Álvarez de Toledo, P., Núñez, F. y Usabiaga, C. (2011b): "El Emparejamiento en las Oficinas Públicas de Empleo Andaluzas: Un Análisis de Duración por Segmentos Laborales", Centro de Estudios Andaluces, mimeo.

Arranz, J.M., García-Serrano, C. y Toharia, L. (2010): "The Influence of Temporary Employment on Unemployment Exits in a Competing Risks Framework", Journal of Labour Research, 31 (1), 67-90.

Bentolila, S., Dolado, J.J. y Jimeno, J.F. (2008): "Does Inmigration Affect the Phillips Curve? Some Evidence for Spain”, European Economic Review, 52 (8), 1398-1423.

Bentolila, S., Michelacci, C. y Suarez, J. (2010): "Social Contacts and Occupational Choice", Economica, 77 (305), 20-45. 
Blanchard, O.J. y Diamond, P. (1992): "The Flow Approach to Labor Markets", American Economic Review, 82 (2), 354-359.

Bover, O., Arellano, M. y Bentolila, S. (2002): "Unemployment Duration, Benefit Duration, and the Business Cycle", Economic Journal, 112 (479), 223-265.

Bover, O. y Gómez, R. (2004): "Another Look at Unemployment Duration: Exit to a Permanent vs. a Temporary Job”, Investigaciones Económicas, 28 (2), 285-314.

Calvó-Armengol, A. (2004): "Job Contact Networks", Journal of Economic Theory, 115 (1), 191-206.

Calvó-Armengol, A. y Matthew, O.J. (2004): "The Effects of Social Networks on Employment and Inequality", American Economic Review, 94 (3), 426-454.

Calvó-Armengol, A. y Matthew, O.J. (2007): "Social Networks in Labor Markets: Wage and Employment Dynamics and Inequality", Journal of Economic Theory, 132 (1), 27-46.

Carrasco, R. (1999): "Transitions To and From Self-Employment in Spain: An Empirical Analysis", Oxford Bulletin of Economics and Statistics, 61 (3), 315-341.

Coles, M.G. y Smith, E. (1998): "Marketplaces and Matching", International Economic Review, 39 (1), 239-254.

Conde-Ruiz, J.I., García, J.R. y Navarro, M. (2009): "Inmigración y Crecimiento Regional en España", FEDEA, Colección Estudios Económicos, Serie Inmigración, nº 09-08.

Congregado, E. y García-Pérez, J.I. (2002): "El Problema del Desempleo en la Economía Andaluza (1990-2001). Análisis de la Transición desde la Educación al Mercado Laboral", Centro de Estudios Andaluces, Documento de Trabajo ${ }^{\circ}$ E2002/17.

Ebrahimy, E. y Shimer, R. (2010): "Stock-Flow Matching", Journal of Economic Theory, 145 (4), 1325-1353.

Elhorst, J.P. (2003): "The Mystery of Regional Unemployment Differentials: Theoretical and Empirical Explanations", Journal of Economic Surveys, 17 (5), 709-748.

Gámez, C. y García-Pérez, J.I. (2004): "Flujos de Trabajadores en el Mercado de Trabajo Andaluz (1978-1999)", Revista Asturiana de Economía, 29, 63-89. 
García-Pérez, J.I. (2006): "Job Separation in a Non-Stationary Search Model: A Structural Estimation to Evaluate Alternative Unemployment Insurance Systems", Journal of Applied Econometrics, 21 (2), 245-272.

Güell, M. y Hu, L. (2006): "Estimating the Probability of Leaving Unemployment Using Uncompleted Spells from Repeated Cross-Section Data", Journal of Econometrics, 133 (1), 307-341.

Güell, M. y Petrongolo, B. (2007): "How Binding are Legal Limits? Transitions from Temporary to Permanent Work in Spain", Labour Economics, 14 (2), 153-183.

Jenkins, S.P. (2005): Survival Analysis, Institute for Social and Economic Research, Lecture Notes.

Jenkins, S.P. y García-Serrano, C. (2004): "The Relationship between Unemployment Benefits and Re-employment Probabilities: Evidence from Spain”, Oxford Bulletin of Economics and Statistics, 66 (2), 239-260.

Jimeno, J.F. y Bentolila, S. (1998): "Regional Unemployment Persistence (Spain, 1976-1994)”, Labour Economics, 5 (1), 25-51.

Mortensen, D.T. (2009): "Island Matching", Journal of Economic Theory, 144 (6), 2336-2353.

Mortensen, D.T. y Pissarides, C.A. (1999): "New Developments in Models of Search in the Labor Market", en Ashenfelter, O.C. y Card, D. (Eds.) (1999): Handbook of Labor Economics, vol. 3C, North-Holland, Amsterdam, 2567-2627.

Petrongolo, B. y Pissarides, C.A. (2001): "Looking Into the Black Box: A Survey of the Matching Function", Journal of Economic Literature, 39 (2), 390-431.

Pissarides, C.A. (2000): Equilibrium Unemployment Theory, The MIT Press, Cambridge (Mass.).

Shimer, R. (2007): "Mismatch", American Economic Review, 97 (4), 10741101.

Shimer, R. (2010): Labour Markets and Business Cycles, Princeton University Press, Princeton.

Usabiaga, C. (2004): El Diferencial de Desempleo Andaluz, Aconcagua, Sevilla. 